\title{
On-Board Computing for Structural Health Monitoring with Smart Wireless Sensors by Modal Identification Using Hilbert-Huang Transform
}

\author{
Ning Wu, ${ }^{1}$ Chengyin Liu, ${ }^{1}$ Yukun Guo, ${ }^{1}$ and Jianhua Zhang ${ }^{2}$ \\ ${ }^{1}$ Harbin Institute of Technology Shenzhen Graduate School, Shenzhen 518055, China \\ ${ }^{2}$ College of Computer Science and Technology, Zhejiang University of Technology, Hangzhou 310023, China
}

Correspondence should be addressed to Chengyin Liu; chengyin.liu08@gmail.com

Received 14 December 2012; Accepted 6 February 2013

Academic Editor: Shengyong Chen

Copyright (c) 2013 Ning Wu et al. This is an open access article distributed under the Creative Commons Attribution License, which permits unrestricted use, distribution, and reproduction in any medium, provided the original work is properly cited.

\begin{abstract}
Smart wireless sensors have been recognized as a promising technology to overcome many inherent difficulties and limitations associated with traditional wired structural health monitoring (SHM) systems. Despite the advances in smart sensor technologies, on-board computing capability of smart sensors has been considered as one of the most difficult challenges in the application of the smart sensors in SHM. Taking the advantage of recent developments in microprocessor which provides powerful on-board computing functionality for smart sensors, this paper presents a new decentralized data processing approach for modal identification using the Hilbert-Huang transform (HHT) algorithm, which is based on signal decomposition technique. It is shown that this method is suitable for implementation in the intrinsically distributed computing environment found in wireless smart sensor networks (WSSNs). The HHT-based decentralized data processing is, then, programmed and implemented on the Crossbow IRIS mote sensor platform. The effectiveness of the proposed techniques is demonstrated through a set of numerical studies and experimental validations on an in-house cable-stayed bridge model in terms of the accuracy of identified dynamic properties.
\end{abstract}

\section{Introduction}

Vibration-based structural health monitoring (SHM) provides valuable information regarding the dynamic characteristics of structures. The identification process consists of measuring vibration responses from structures and analyzing the measured data to build a numerical model of the structure. Traditionally, the vibration responses are obtained using centralized data acquisition systems with wired sensors. However, wired sensors have proven to be difficult to implement, particularly for dense deployment of sensors on largescale civil infrastructures due to the long setup time, the difficulties in cabling, and the high cost in equipments.

Smart wireless sensors are more flexible than traditional wired sensors in communication, and their performance in data processing is also better [1-3]. A smart sensor is equipped with an independent on-board microprocessor that can be used for digital signal processing, self-diagnosis, self-identification, and self-adaptation, and the smart sensor platform is battery powered with reliable wireless communication technology.

Smart sensors provide a promising alternative to the traditional wired sensor systems, and there have been many successful applications. Spencer et al. [4] defined smart sensors with mainly four features: (i) on-board computing capability, (ii) small size, (iii) wireless communication, and (iv) low cost. Lynch and Loh [5] reviewed over 150 papers on wireless sensors for SHM with research conducted at more than 50 institutes worldwide. SHM applications with smart sensors have been studied using both scale models and full-scale structures, respectively. Recent studies [6-11] have demonstrated the potential of applying smart sensors to monitor large-scale civil infrastructure using a dense array of sensors. However, the power consumption and long-term reliability of smart sensors are still expected to be improved. Many different methods have been proposed for power harvesting 
TABLE 1: IRIS features.

\begin{tabular}{lcccccc}
\hline Processor & $\begin{array}{c}\text { Clock speed } \\
(\mathrm{MHz})\end{array}$ & $\begin{array}{c}\text { Memory } \\
(\mathrm{KB})\end{array}$ & $\begin{array}{c}\text { SRAM } \\
(\mathrm{KB})\end{array}$ & $\begin{array}{c}\text { Program flash } \\
(\mathrm{KB})\end{array}$ & $\begin{array}{c}\text { Radio } \\
(\mathrm{Hz})\end{array}$ & $\begin{array}{c}\text { Data rate } \\
(\mathrm{kbp} / \mathrm{sec})\end{array}$ \\
\hline $\begin{array}{l}\text { ATMegall } \\
281 \mathrm{~V}\end{array}$ & 8 & 128 & 8 & 512 & $2.4 \mathrm{G}$ & 250 \\
\hline
\end{tabular}

of sensors, for example, solar power and vibration power $[6,12-14]$, but the system development is too complicated. One way to alleviate this problem is to reduce the number of communication cycles, since, in statistics, communication consumes most of the battery energy.

The performance of data communication in wireless smart sensor networks (WSSNs) is based on the data acquisition and processing schemes within the WSSNs. A singlesensor node which generates 16-bit vibration data along three axes at 500 samples per second can easily consume onefourth of the nominal data rate of the IEEE 802.15.4 lowpower radio. A large structure typically comprises hundreds of members and will require at least two triaxial sensors measuring accelerations at each end of every member, so that the damaged members can be detected. Clearly, transferring raw sensor data in a continuous mode for a traditional central data repository is not an efficient way for the WSSNs. Therefore, decentralized approaches have been introduced to process the sensor data within the network before transmitting it to a central computer [15]. The key challenge here is how to adapt the existing SHM signal-processing techniques to perform as much data reduction within the network as possible.

The extraction of the modal information, such as modal frequencies and mode shapes from sensor data is very important for the assessment of the structural performance and the calibration of the analytical design model [16]. In an attempt to merge the modal identification methods into a state-ofthe-art wireless sensing paradigm, the Peak Picking (PP) method and the Frequency Domain Decomposition (FDD) technique [17] are modified for use within a distributed (i.e., decentralized) wireless sensing network [18]. While the amount of data wirelessly transferred in the network is significantly reduced when using these independent processing approach, modal identification methods have been addressed with some drawbacks in terms of their sensitivity to environmental effects such as temperature and moisture and nonlinearities [19].

A time-series analysis, known as the Hilbert-Huang transform (HHT), is recently introduced by Huang et al. [20] and has received considerable attention in structural health monitoring applications. The HHT is an adaptive signalprocessing technique that produces signal decomposition in both time and frequency domains, providing instantaneous frequencies, phase, and damping for extracting damage sensitive features from the processed and decomposed data [2124].

In order to realize data aggregation and modal identification based on HHT, this paper proposes a new distributed SHM scheme for the implementation in the intrinsically decentralized computing environment in WSSNs. In this study, a laboratory bridge model is built and tested on a commercial off-the-shelf WSN platform. The identification of the modal properties for vibration signals using the embedded HHT method on smart sensors is examined. The results from both simulation and experiments show that the proposed method achieves higher accuracy for identifying modal characteristics. The on-board computing features of the proposed system are also proven to be tolerant to environmental fluctuations in temperature and noise levels.

In the next section of this paper, the distributed SHM scheme will be introduced, and the simulation and experimental results will be shown in Sections 3 and 4. Finally, a conclusion will be made.

\section{Background}

2.1. Wireless Smart Sensor Node. As it is shown in Figure 1, the IRIS mote wireless sensor was developed by the researchers at the University of California, Berkeley [25], and it is a smart sensor platform designed for data intensive applications. The IRIS is an open hardware and software platform for smart sensing, and it consists of a plug-in sensor boards, a processor, a transceiver, and an AA battery pack. The specifications are shown in Table 1 . The processor speed may be scaled based on the application requirement, and thereby maintaining its power usage efficiency.

A variety of sensor boards for the mote are available in the market. MTS400 sensor board from Crossbow Technology is one of the most popular sensors used in research [26], and it is implemented with acceleration, light, pressure, and temperature sensors, as shown in Table 2. In this paper, an IRIS with an MTS400 sensor board will be used in the decentralized SHM system.

The operating system used on the IRIS for this research is TinyOS, which is a distributed, open-source operating system [27]. TinyOS supports large-scale, self-configuring sensor networks, and its functions include radio messaging, message hopping from mote to mote, low-power modes, sensor measurements, and signal processing. NesC is used as the programming language for TinyOS. This operating system has a small memory footprint and is, therefore, suitable for smart sensors with limited resource. TinyOS also has a large user community and many successful smart sensor applications.

2.2. Hilbert-Huang Transform Algorithm. In 1998, Huang et al. [20] introduced a new adaptive signal-processing method, referred to as the Hilbert-Huang transform (HHT), suitable for the requirements for processing linear, nonlinear, stationary, and nonstationary signals. The HHT consists of 
TABLE 2: Sensors on MTS400.

\begin{tabular}{lccc}
\hline Sensor & Type & Measurement range & Resolution \\
\hline Acceleration & Analog devices ADXL202JE & $\pm 2(\mathrm{~g})$ & $2 \mathrm{mg}(60 \mathrm{~Hz})$ \\
Pressure & Intersema MS5534AM & $300-1100 \mathrm{mbar}$ & $0.1 \mathrm{mbar}$ \\
Light & TAOSTSL2550D & $400-1000 \mathrm{~nm}$ & None \\
Temperature & Sensirion SHT11 & $0-100 \% \mathrm{RH} ;$ & $0.03 \% \mathrm{RH}$ \\
\hline
\end{tabular}

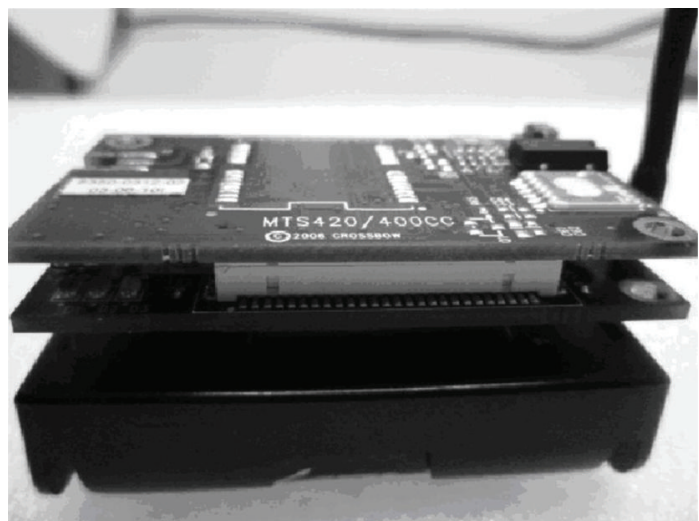

(a)

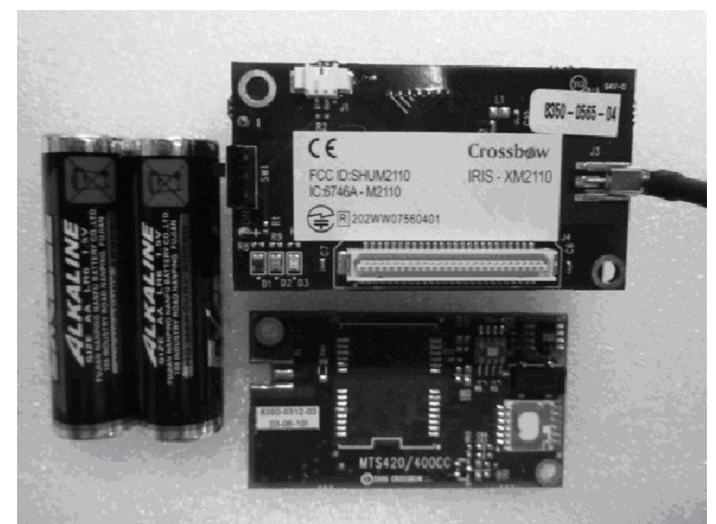

(b)

FIGURE 1: IRIS mote with MTS400 board (a) and its components (b).

two main parts: (i) EMD and (ii) Hilbert transform. The EMD method decomposes a real signal into a collection of simpler modes or intrinsic mode functions (IMFs), which are basically associated with the energy of the signal at different timescales and contain important data characteristics [20]. The Hilbert transform, when applied to IMFs, will produce an energy-frequency-time distribution of the data, known as a Hilbert spectrum. In general, the decomposition resulting from HHT is well localized in the time-frequency domain and reveals important information within data.

The EMD extracts the IMFs by applying a sifting process to a given time signal, and two cubic splines are fitted through the signal's local maxima and minima in order to produce an upper and lower envelope, respectively. The average of these two splines is then subtracted from the original signal. The resultant signal is subsequently treated and repeated as the original time signal. The first IMF component contains the shortest period component of the signal. In order to derive other IMFs, the first IMF is removed from the signal, the residue is considered as the new signal, and the sifting process is performed to obtain the second IMF component. This procedure is repeated for all subsequent residues to derive the longer period components. After extracting all IMFs, the original signal is decomposed into $n$ empirical modes, $c_{i}$, and a residue, $r_{n}$, which can either be the mean trend or a constant. This can be represented by the following equation:

$$
x(t)=\sum_{i=1}^{n} C_{i}(t)+r_{n}(t) .
$$

After extraction of IMFs through the sifting process, the Hilbert transform can be applied to each IMF in order to obtain the amplitude, phase, and frequency information, respectively. For a given $\operatorname{IMF} C_{i}(t) \equiv x(t)$, the Hilbert transform $y(t)$ is defined as the convolution of $x(t)$ and $1 /(\pi t)$. The mathematical form of Hilbert transform is expressed as

$$
H[x(t)]=y(t)=\frac{1}{\pi} P \int_{-\infty}^{+\infty} \frac{x(\tau)}{t-\tau} d \tau,
$$

where $P$ indicates that the integral is to be considered as a Cauchy principal value, which prevents a possible singularity at $t=\tau$ and $\pm \infty$. When the Hilbert transform is applied to $x(t)$, the magnitude is kept unchanged but the phase of all frequency components is shifted by $\pi / 2$. Using the Hilbert transform, an analytic signal $z(t)$ can be defined as the complex conjugate of $x(t)$ and its Hilbert transform $y(t)$ [20],

$$
\begin{gathered}
z(t)=x(t)+i y(t)=a(t) e^{i \theta(t)}, \\
a(t)=\sqrt{x^{2}(t)+y^{2}(t),} \\
\theta(t)=\arctan \left(\frac{y(t)}{x(t)}\right),
\end{gathered}
$$

where $a(t)$ and $\theta(t)$ are called the amplitude and the instantaneous phase function of $x(t)$, respectively. Mathematically, the above polar expression provides the best local fit for any signal $x(t)$ with varying amplitude and phase [20]. Because the IMFs are symmetric with respect to zero mean, the instantaneous phase increases monotonically as a function of time.

In summary, the processing of vibration signals by EMD coupled with the Hilbert transform reveals significant fundamental information hidden in the signals for detecting damage sensitive features in structural health monitoring. 
TABLE 3: Modal frequencies obtained from on-board and offline computing in numerical example.

\begin{tabular}{lccc}
\hline Mode & Frequency of on-board result & Frequency of offline result & Error \\
\hline First order & $1.91 \mathrm{~Hz}$ & $1.98 \mathrm{~Hz}$ & $3.60 \%$ \\
Second order & $3.82 \mathrm{~Hz}$ & $3.76 \mathrm{~Hz}$ & $1.50 \%$ \\
\hline
\end{tabular}

TABLE 4: Experimental cable-stayed bridge model mechanical characteristics.

\begin{tabular}{|c|c|c|c|c|c|c|}
\hline Element & Cross section & Material & Density $\left(\mathrm{kg} / \mathrm{m}^{3}\right)$ & Modulus (GP) & Poisson ratio & Dimension (m) \\
\hline Middle tower & Box $\square$ & Aluminum alloy & 2700 & 68.6 & 0.34 & $3.1 \times 0.1375 \times 0.095$ \\
\hline Side tower & Box $\square$ & Aluminum alloy & 2700 & 68.6 & 0.34 & $1.9 \times 0.12 \times 0.075$ \\
\hline Deck & Separate box & Aluminum alloy & 2700 & 68.6 & 0.34 & $15.2 \times 0.82 \times 0.07$ \\
\hline Cable & Circle $\bigcirc$ & High-strength steel wire & 7850 & 200 & 0.30 & varies \\
\hline
\end{tabular}

Depending on the structure being studied, there are various types of data extracted from the HHT and EMD. This data include intrinsic mode functions, instantaneous frequency, phase, amplitude, and damping. The next section discusses the implementation of this strategy on smart sensor platform.

2.3. Smart Sensor System Realization. The implementation of the decentralized approach is composed of a modal identification algorithm and middleware services. Major numerical routines utilized in this approach include empirical modal decomposition (EMD), quick sort, and Hilbert transform. These functions are either developed from scratch or adapted from functions previously written in $\mathrm{C}$ language. The performance of these functions is examined on the IRIS system. Middleware services for the SHM include data aggregation, reliable communication, and synchronized sensing. These techniques are coded as $\mathrm{C}$ functions and compatible with TinyOS.

The time synchronization and reliable data delivery mechanisms in the framework can be implemented, respectively, by using suitable existing techniques; however, this issue is beyond the scope of this paper. The main focus of this paper is on the design of distributed algorithms for WSN-based SHM. When the synchronized sensing is completed, the data acquired will be processed immediately. The measured acceleration time histories and the intermediate processed results, such as the structural modal parameters, are sent back to the back-end server, where damage alarm or other applications will be executed.

\section{Numerical Studies}

The objective of the numerical studies in this section is to investigate the performance of the proposed distributed modal identification method for structural health monitoring based on the embedded processing capability of smart sensors. An original sine function signal, $y=100 * \sin (24 * t)+$ $100 * \sin (12 * t)$, for simulation is selected in order to identify its dominant frequencies.

Cheraghi et al. [28, 29] suggested a band-pass filtering scheme in order to retain the interested frequencies only. In the present study, the signal is filtered with various bands according to the former Fourier transformation results. The first range is from 1 to $1.5 \mathrm{~Hz}$, and the second range is from 3.5 to $5 \mathrm{~Hz}$. The amplitudes at other frequency ranges are much lower than the bands mentioned before.

Figure 2 illustrates the IMF and phase angle obtained from on-board computing and offline computing, respectively, for the first frequency case. From Figure 2 it can be seen that for all frequency cases, the IMF and phase calculated on IRIS sensor have very good agreement with those obtained by offline calculation method. After resolving the phase by the least square method, the frequencies in this example as listed in Table 3 can be obtained. The first and second frequencies measured from the on-board computing are $1.91 \mathrm{~Hz}$ and $3.82 \mathrm{~Hz}$, respectively. In contrast to $1.98 \mathrm{~Hz}$ and $3.76 \mathrm{~Hz}$ from the offline computing, the errors are $3.6 \%$ and $1.5 \%$ for the first and second frequency, respectively. The major influencing factors resulting in these errors may be due to the limited $\mathrm{CPU}$ power, accumulated errors, and data package lagging in numerical processing. However, these errors are small and ignorable in engineering applications. In general, it can be concluded that the on-board computing technique of smart sensor based on HHT algorithm is reliable, and the smart characteristics of wireless sensor could be used for the SHM purpose.

\section{Validation Test and Result Analysis}

In an attempt to further investigate the onboard computing capabilities in smart sensors, the same methodology is applied to examine an in-house cable-stayed bridge model built in a laboratory. The vertical vibration signals of the bridge model in the tests is measured by the IRIS installed along the bridge deck. White noise loads are applied through two magnetic vibration generators underneath the bridge deck. The same test is carried out for 15 times with the sampling frequency of $50 \mathrm{~Hz}$, and the duration of each record is about $1 \mathrm{sec}$.

4.1. Test Structure. The test structure represents a reducedscale model of Shandong Binzhou Yellow River cable-stayed Bridge (see Figure 3). The two-span bridge model is mainly made of aluminum. Effective stiffness criterion is utilized in the reduced-scale dynamic-elastic bridge model design. Additional masses are added so the bridge model which gives 


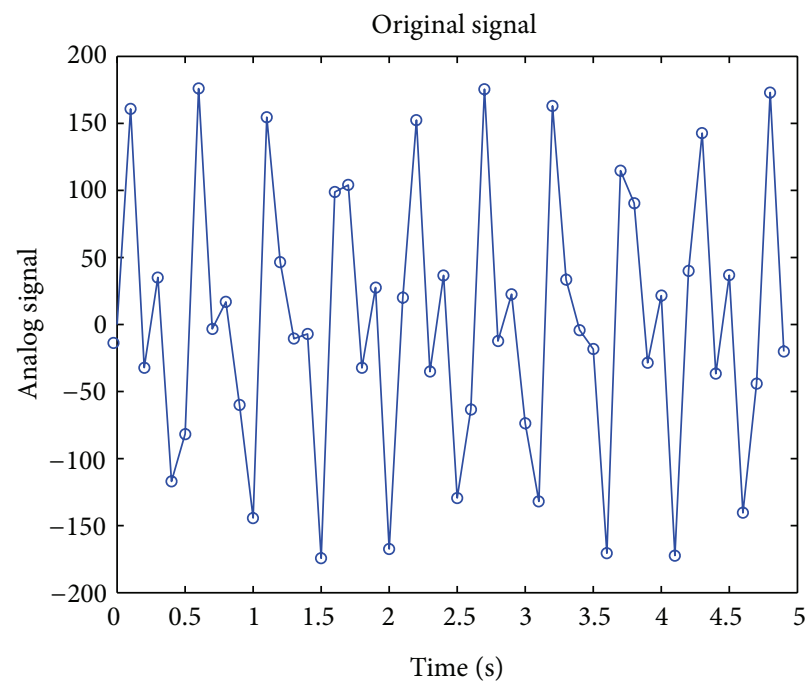

(a) On-board original signal

Filtered signal

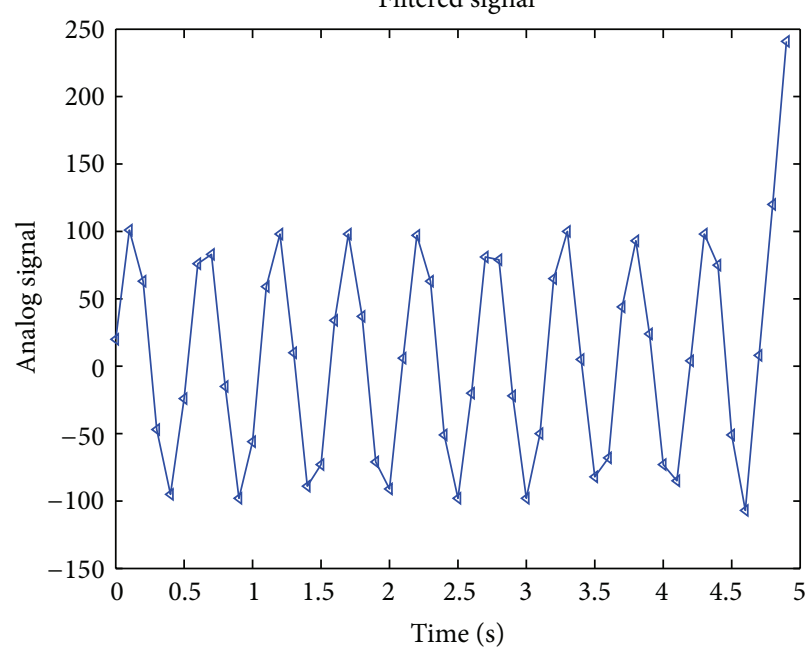

(c) On-board band-pass filtered signal

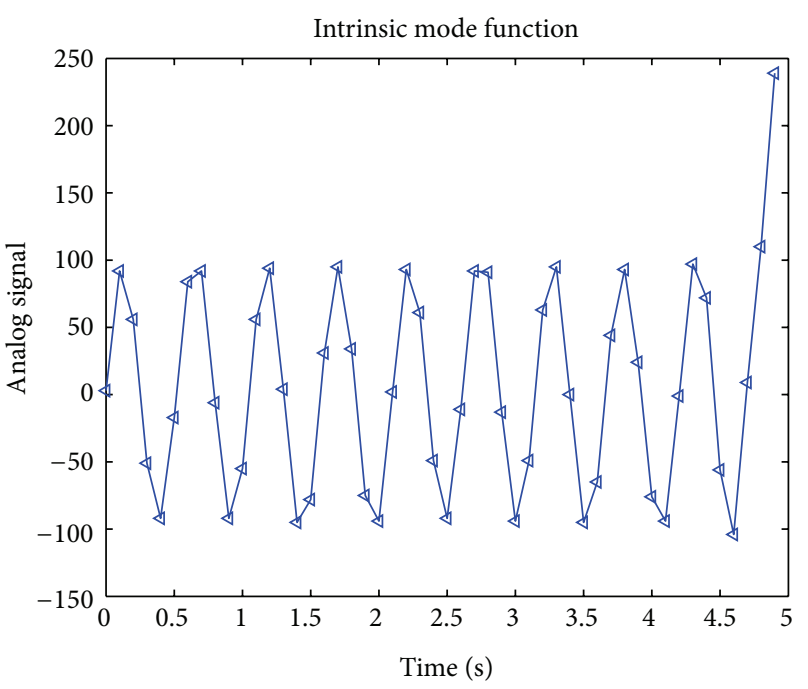

(e) On-board IMF

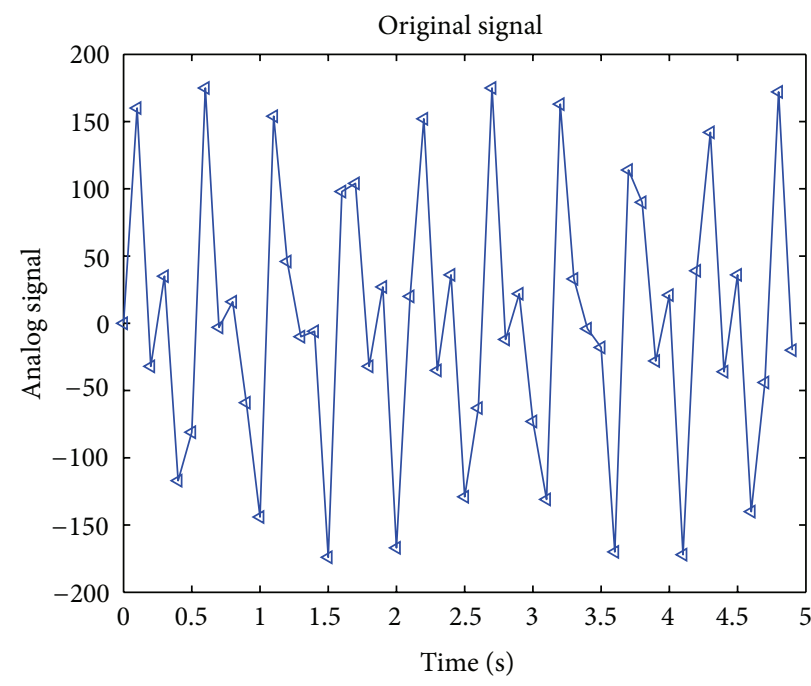

(b) Offline original signal

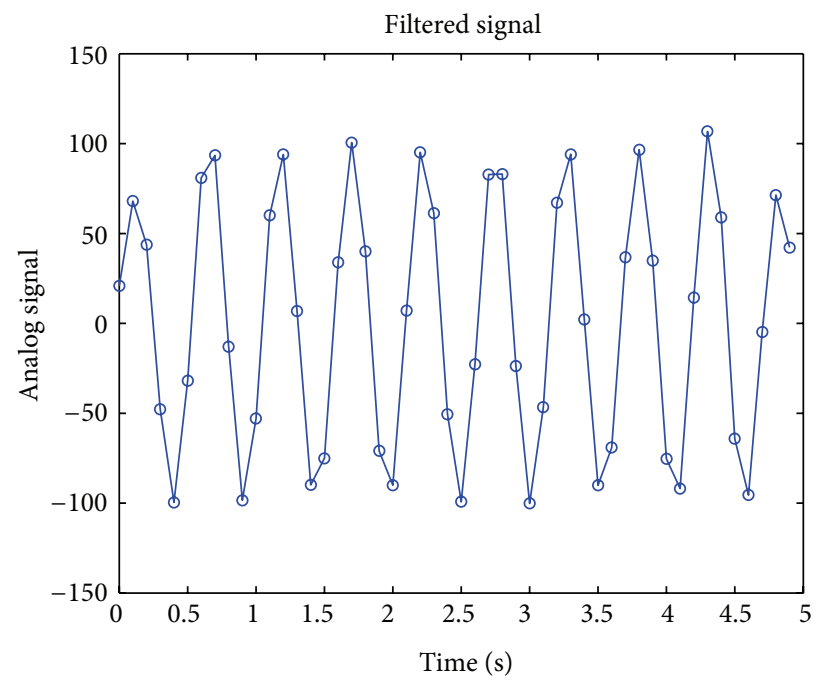

(d) Offline band-pass filtered signal

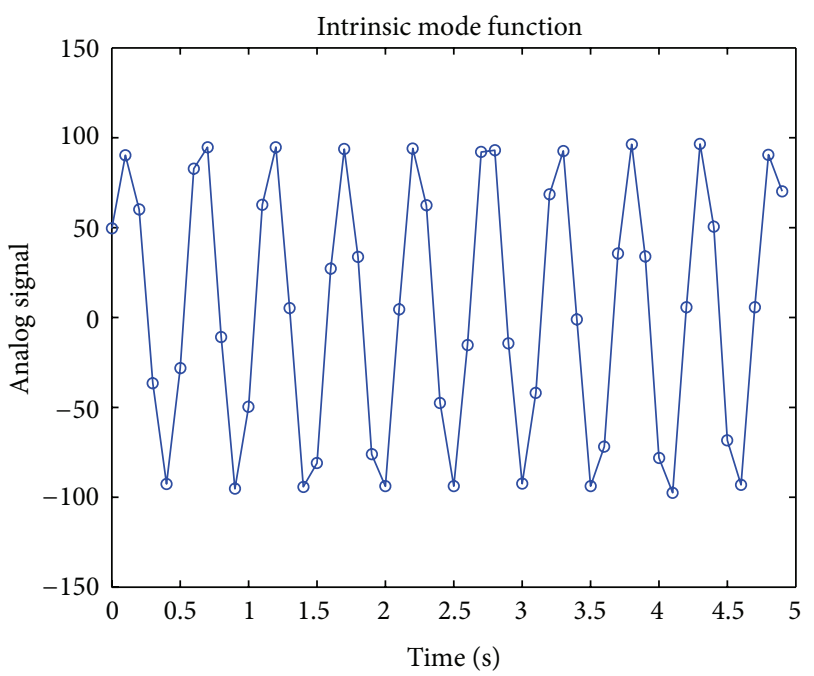

(f) Offline IMF

Figure 2: Continued. 


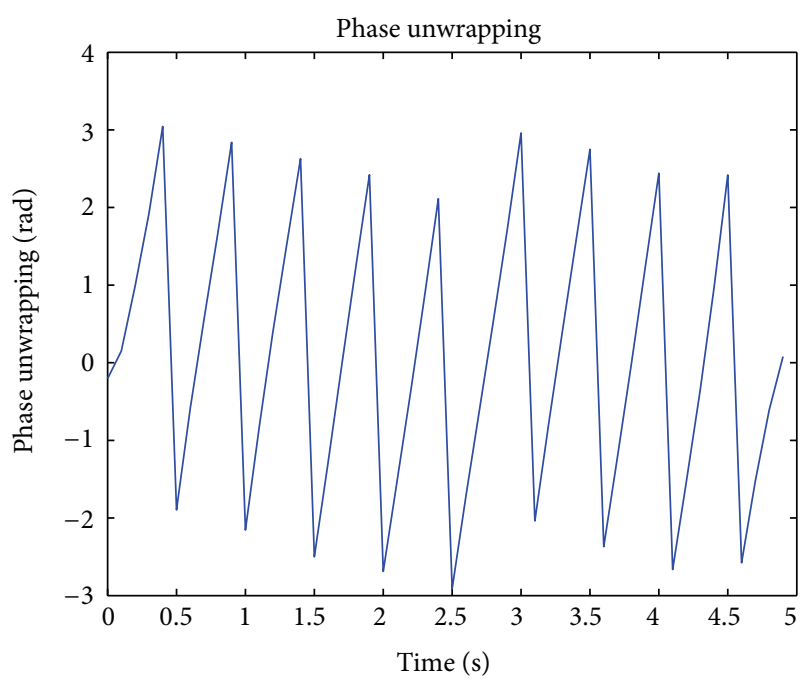

(g) On-board phase unwrapping

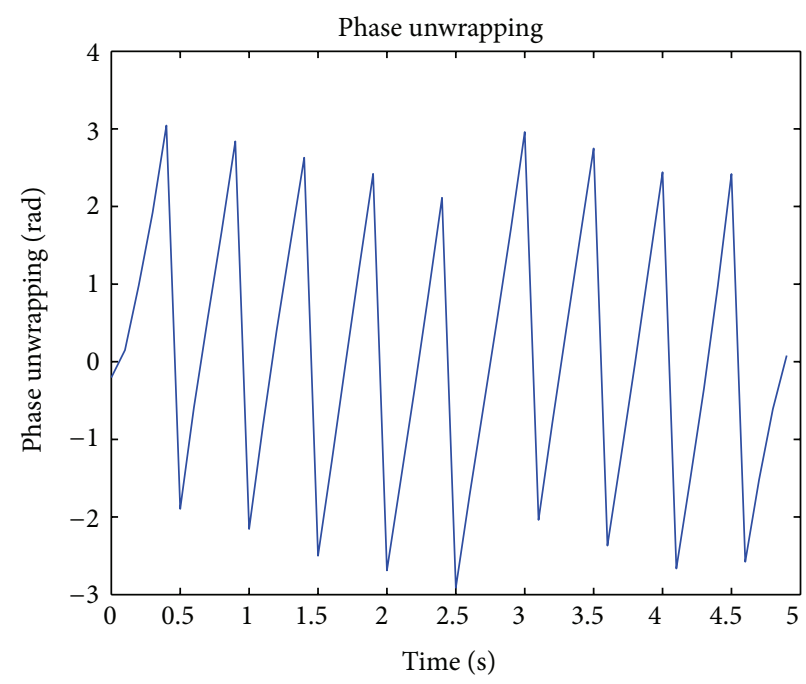

(h) Offline phase unwrapping

FIGURE 2: Numerical HHT algorithm evaluated from on-board computing and offline computing for the first frequency case.

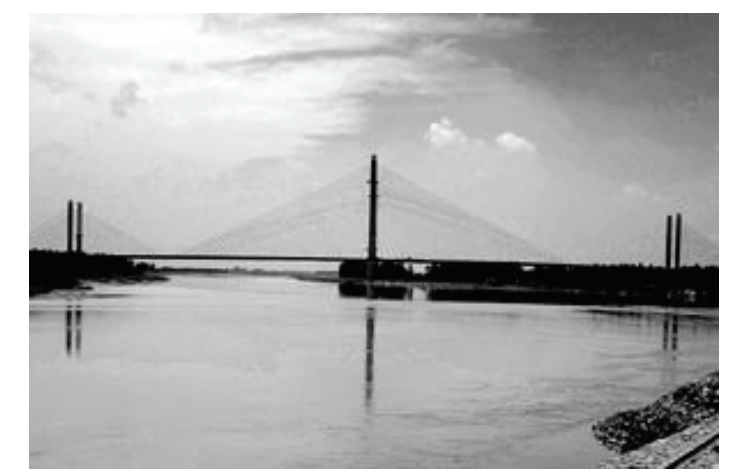

FIgURE 3: Photo of Binzhou Yellow River cable-stayed bridge.

similar dynamic characteristics within the range of the prototype bridge. The bridge model is eventually built in a ratio of 1: 40 to the prototype bridge considering lab conditions and costs (see Figure 4). Table 4 lists the specific mechanical parameters of the bridge model. Several experiments have been previously done with various configurations of this structure [30] based on the similarity theory, in order to show that the reduced-scale bridge model with added masses has a good degree of similarity with the prototype bridge.

4.2. Finite Element Analysis. In order to verify the accuracy of testing results and provide dominant frequency ranges, a full three-dimensional finite element model is developed using ANSYS. The geometry and member details of the model are based on the design data of the laboratory test bridge model. The main structural members are composed of stay cables, floor beams, and towers, all of which are discreterized by different finite element types.

The modeling of stay cable can be realized in ANSYS by employing the $3 \mathrm{D}$ tension-only truss elements (LINK8) and utilizing its stress-stiffening capability. Each stay cable

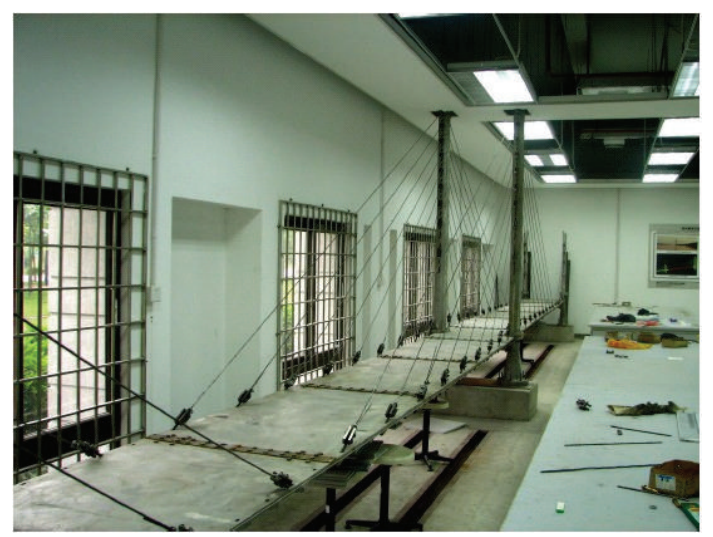

FIGURE 4: Photo of the reduced-scale laboratory bridge model.

is modeled by one element, which results in 60 tension-only truss elements in the model. Two steel main girders are modeled as the 3D elastic beam elements (BEAM4) for simplicity, since they are the structural members possibly subjected to tension, compression, bending, and torsion. There are in total 508 elements of this type. Towers consist of both equivalent and variable sections so that they are discreterized by BEAM188 elements with a total number of 140 . All piers and platforms are modeled by the solid elements (SOLID45), of which there are 852 elements. The diaphragms are modeled by 80 beam elements (BEAM44). In addition, 210 concentrated mass elements (MASS21) are used to include the mass of equilibrium blocks, parapet, and anchors that are nonstructural members.

The complete model consists of 1371 nodes and 1500 elements resulting in 8202 active degrees of freedom (DOFs). The model represents the bridge in its current as-built configuration and structural properties. The first three vertical bending modal shapes of bridge model from FEA are shown 
TABLE 5: On-board computing, offline computing, and FE results.

\begin{tabular}{|c|c|c|c|c|}
\hline Mode & Frequency from on-board computing & Frequency from offline computing & Errors & FE result \\
\hline First order & $4.64 \mathrm{~Hz}$ & $4.52 \mathrm{~Hz}$ & $2.6 \%$ & $4.23 \mathrm{~Hz}$ \\
\hline Second order & $8.69 \mathrm{~Hz}$ & $8.66 \mathrm{~Hz}$ & $0.27 \%$ & $10.92 \mathrm{~Hz}$ \\
\hline Third order & $10.47 \mathrm{~Hz}$ & $10.52 \mathrm{~Hz}$ & $0.49 \%$ & $12.16 \mathrm{~Hz}$ \\
\hline
\end{tabular}

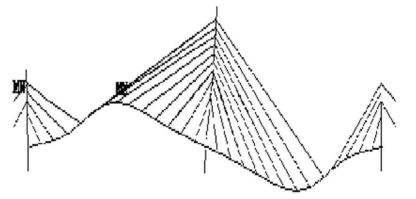

(a) 1st Vertical Bending

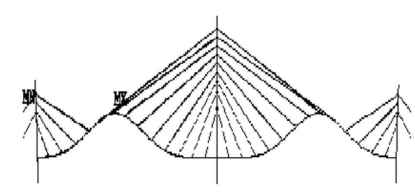

(b) 2nd Vertical Bending

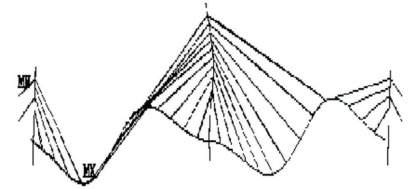

(c) 3rd Vertical Bending

Figure 5: The operational deflection shapes for the first three fundamental modes of vibration.

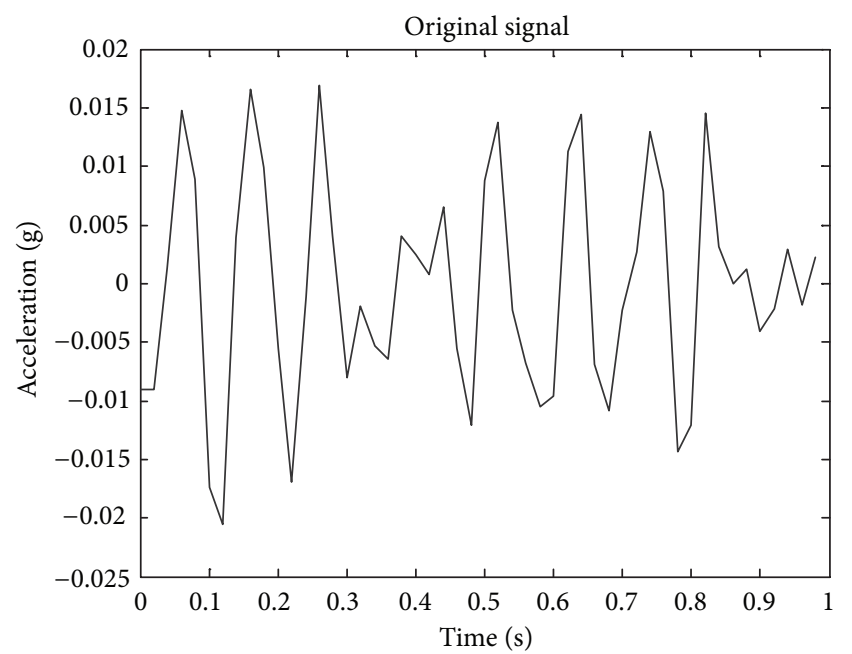

Figure 6: Typical acceleration signal of wireless sensor from testing.

in Figure 5, and their corresponding natural frequencies are listed in Table 5.

4.3. Testing Results. After obtaining the encouraging results from $\mathrm{FE}$ analysis, the application of the proposed approach is examined experimentally on the cable-stayed bridge model. The band-pass filtering used in this experimental test should be carefully implemented in order to take into account the frequency components that are only sensitive to the bridge model. According to the afore mentioned FE analysis, it is concluded that band-pass filters with the band-pass frequency of $3.5-5.0 \mathrm{~Hz}, 8.0-9.5 \mathrm{~Hz}$, and 10.0-11.0 Hz (corresponding to the first three bridge vibration modes), respectively, can give rise to the best resolution for modal parameter identification.

Typical experimental results of this investigation are presented in Figures 6 and 7 for the first natural frequency case, respectively. Figure 6 shows the original experimental acceleration signal from a typical wireless sensor node. Figure 7 shows the band-pass filtered signal and the IMF along with phase obtained both by on-board computing and offline computing for the first natural frequency case. It can be found that the estimated IMF obtained by the on-board computing method is in excellent agreement with the offline computing counterpart. Based on the IMF and phase values, we can calculate the natural frequency based on the least square method. Similar to the first natural frequency, the second and third natural frequency can be obtained using the same procedure. The natural frequencies obtained from the on-board computing are shown in Table 5, and they are compared with the results from the offline computation and FE analysis. For all three cases, the largest relative error of natural frequency obtained from the on-board computing to that from the offline computing is only $2.6 \%$ (in the first case). It can be seen that the proposed embedded method has achieved the modal parameters comparable with those obtained using the traditional method, but the energy consumption has greatly been reduced.

\section{Conclusions}

In this study, an application of the HHT-based decentralized data processing approach is proposed based on smart wireless sensors, and it has been verified with both simulation and experiment. The basic concept is to analyze the structural vibration signals through the EMD and HHT, taking advantage of on-board computing capability of smart sensor. The proposed approach is programmed and embedded into the data processing module of a smart sensor. The performance of decentralized HHT is assessed in terms of accuracy of the identified modal properties of the test structure.

A laboratory cable-stayed bridge model is employed to investigate the performance of decentralized HHT on the IRIS wireless sensor platform. The offline data processing approach is selected as a reference for numerical comparison, and finite element analysis has been introduced to provide necessary modal information of the structure. Results from both simulation and experiment show that in all dominant frequency cases the smart sensor implemented with on-board HHT algorithm can identify the modal parameters such as the natural frequency at the same resolution as the traditional offline approach. More importantly, the proposed method 


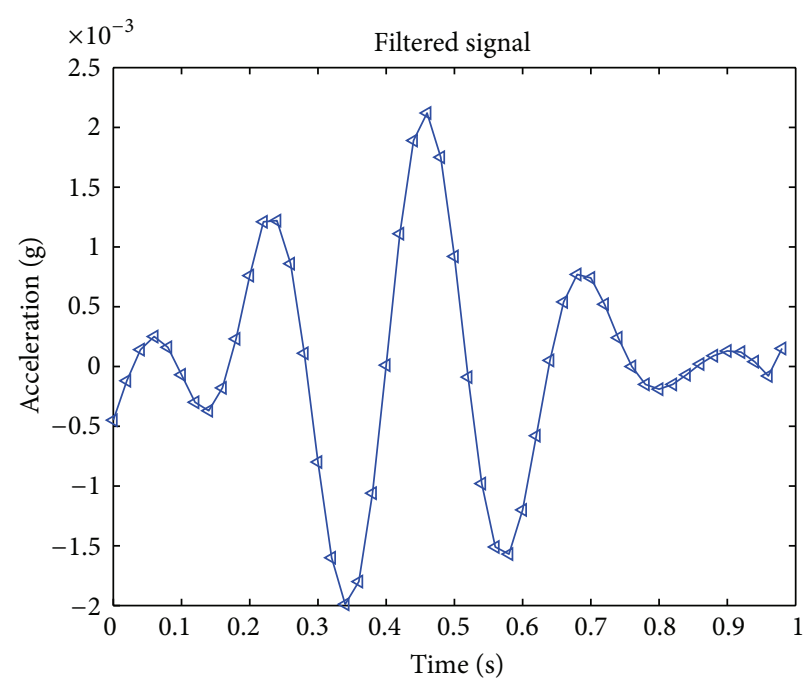

(a) On-board band-pass filtered signal

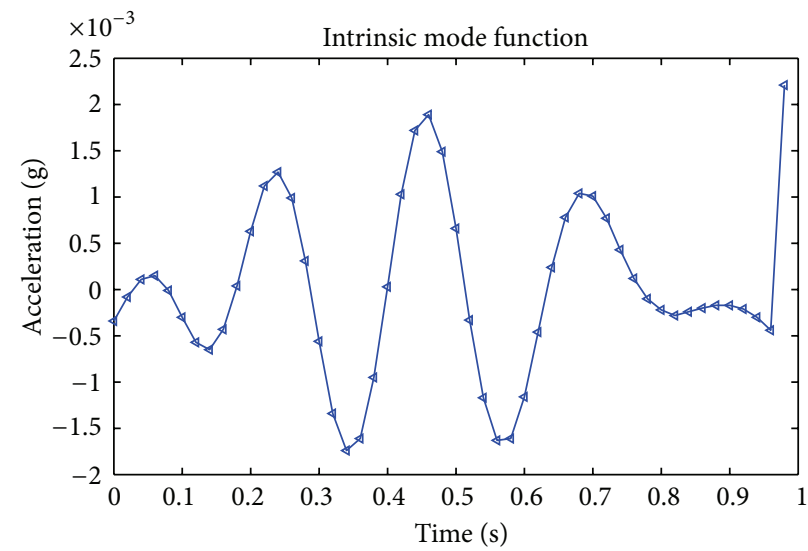

(c) On-board IMF

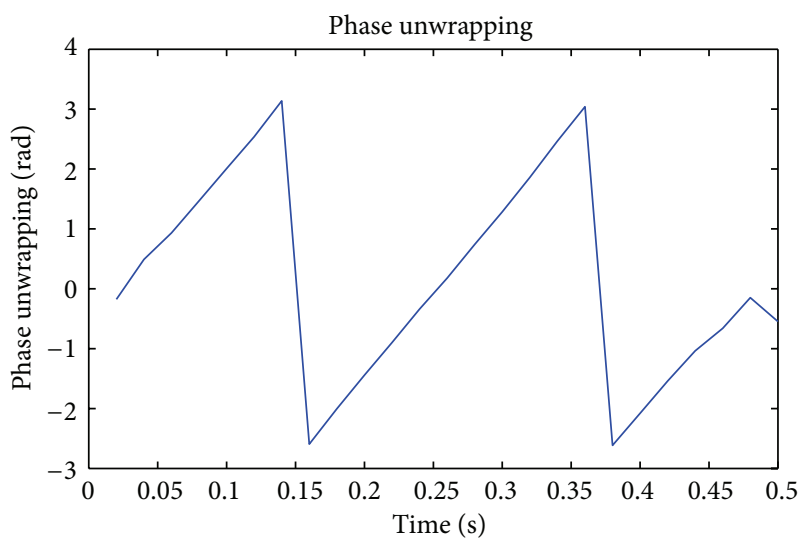

(e) On-board Phase-unwrapping

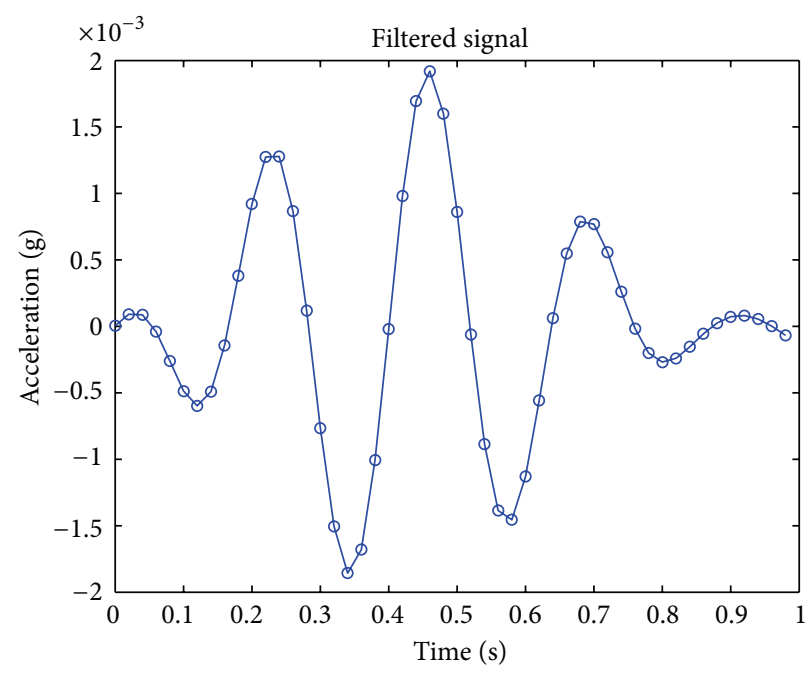

(b) Offline band-pass filtered signal

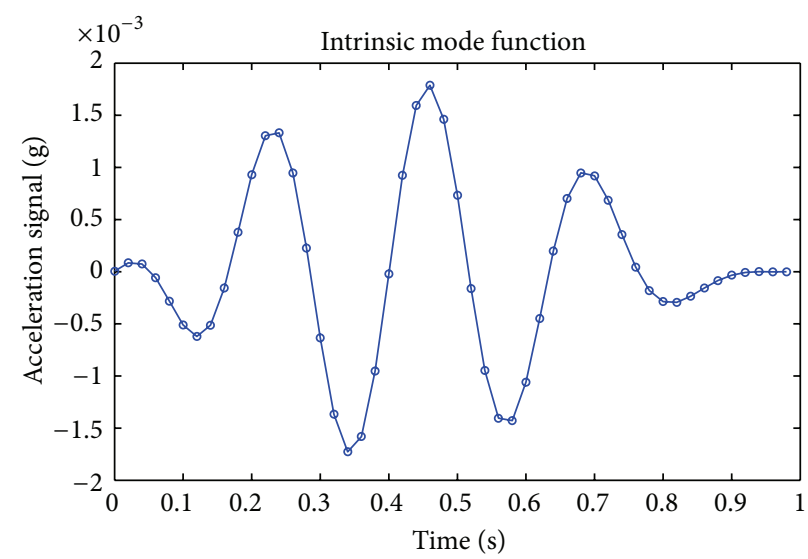

(d) Offline IMF

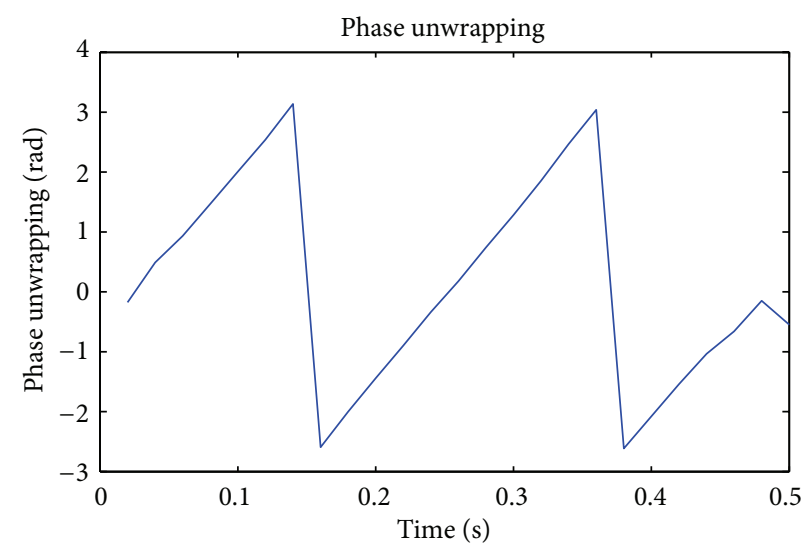

(f) Offline Phase-unwrapping

FIGURE 7: Experimental HHT algorithm evaluated from on-board computing and offline computing for the first frequency case.

could locally compute these coefficients and transmit them to the central location, instead of transmitting the entire data. Therefore, this method increases the efficiency in wireless communication for real applications of SHM.

Overall, the proposed modal identification technique in this study is a low cost and effective tool for SHM. Based on the experimental results, we can see that there is a great potential to develop a practical and reliable modal identification procedure for WSN-based structural health monitoring. Despite the successful implementation of the HHT and EMD methods on the laboratory scale, some issues must yet be taken into account when considering real-world 
applications, where a large number of sensors will be densely deployed over a large structure.

\section{Acknowledgments}

Financial supports from the National Science Foundation of China (no. 51108129) and Guangdong Science and Technology Major Project (no. 2012A080104014) to initiate this research project are greatly acknowledged.

\section{References}

[1] H. Shi, W. Wang, N. M. Kwok, and S. Y. Chen, "Game theory for wireless sensor networks: a survey," Sensors, vol. 12, no. 7, pp. 9055-9097, 2012.

[2] C. Cattani, S. Chen, and G. Aldashev, "Information and modeling in complexity," Mathematical Problems in Engineering, vol. 2012, Article ID 868413, 4 pages, 2012.

[3] S. Chen, W. Huang, C. Cattani, and G. Altieri, "Traffic dynamics on complex networks: a survey," Mathematical Problems in Engineering, vol. 2012, Article ID 732698, 23 pages, 2012.

[4] B. F. Spencer Jr., M. E. Ruiz-Sandoval, and N. Kurata, "Smart sensing technology: opportunities and challenges," Journal of Structural Control and Health Monitoring, vol. 11, no. 4, pp. 349368, 2004.

[5] J. P. Lynch and K. J. Loh, "A summary review of wireless sensors and sensor networks for structural health monitoring," The Shock and Vibration Digest, vol. 38, no. 2, pp. 91-128.

[6] S. Jang, H. Jo, S. Cho et al., "Structural health monitoring of a cable-stayed bridge using smart sensor technology: deployment and evaluation," Smart Structures and Systems, vol. 6, no. 5-6, pp. 439-459, 2010.

[7] S. Cho, H. Jo, S. Jang et al., "Structural health monitoring of a cable-stayed bridge using wireless smart sensor technology: data analyses," Smart Structures and Systems, vol. 6, no. 5-6, pp. 461-480, 2010.

[8] Y. Nitta, T. Nagayama, B. F. Spencer Jr., and A. Nishitani, "Rapid damage assessment for the structures utilizing smart sensor MICA2 MOTE," in Proceedings of the 5th International Workshop on Structural Health Monitoring, pp. 283-290, Standford, Calif, USA, 2005.

[9] Y. Gao, B. F. Spencer Jr., and M. Ruiz-Sandoval, "Distributed computing strategy for structural health monitoring," Structural Control and Health Monitoring, vol. 13, no. 1, pp. 488-507, 2006.

[10] J. A. Rice and B. F. Spencer Jr., "Flexible smart sensor framework for autonomous full-scale structural health monitoring. Newmark Structural Engineering Laboratory (NSEL)," Report Series no. 18, University of Illinois at Urbana-Champaign, Urbana, Ill, USA, 2009, http://hdl.handle.net/2142/13635.

[11] N. A. Tanner, J. R. Wait, C. R. Farrar, and H. Sohn, "Structural health monitoring using modular wireless sensors," Journal of Intelligent Material Systems and Structures, vol. 14, no. 1, pp. 4356, 2003.

[12] S. Castellucci, M. Carlini, M. Guerrieri, and T. Honorati, "Stability and control for energy production parametric dependence," Mathematical Problems in Engineering, vol. 2010, Article ID 842380, 21 pages, 2010.

[13] M. Li, W. Zhao, and S. Chen, "mBm-based scalings of traffic propagated in internet," Mathematical Problems in Engineering, vol. 2011, Article ID 389803, 21 pages, 2011.
[14] M. Rahimi, H. Shah, G. S. Sukhatme, J. Heideman, and D. Estrin, "Studying the feasibility of energy harvesting in a mobile sensor network," in Proceedings of the IEEE International Conference on Robotics and Automation (ICRA '03), pp. 19-24, September 2003.

[15] T. Nagayama, S. H. Sim, Y. Miyamori, and B. F. Spencer, "Issues in structural health monitoring employing smart sensors," Smart Structures and Systems, vol. 3, no. 3, pp. 299-329, 2007.

[16] S. Cho, C. -B. Yun, J. P. Lynch, A. Zimmerman, B. Spencer Jr., and T. Nagayama, "Smart wireless sensor technology for structural health monitoring of civil structures," Journal of Steel Structures, vol. 81, pp. 267-275, 2004.

[17] R. Brincker, L. Zhang, and P. Andersen, "Modal identification of output-only systems using frequency domain decomposition," Smart Materials and Structures, vol. 10, no. 3, pp. 441-445, 2001.

[18] A. T. Zimmerman, M. Shiraishi, R. A. Swartz, and J. P. Lynch, "Automated modal parameter estimation by parallel processing within wireless monitoring systems," Journal of Infrastructure Systems, vol. 14, no. 1, pp. 102-113, 2008.

[19] D. Pines and L. Salvino, "Structural health monitoring using empirical mode decomposition and the Hilbert phase," Journal of Sound and Vibration, vol. 294, no. 1-2, pp. 97-124, 2006.

[20] N. E. Huang, Z. Shen, S. R. Long et al., "The empirical mode decomposition and the Hilbert spectrum for nonlinear and non-stationary time series analysis," The Royal Society of London Series A, vol. 454, no. 1971, pp. 903-995, 1998.

[21] S. Y. Chen, H. Tong, Z. Wang, S. Liu, M. Li, and B. Zhang, "Improved generalized belief propagation for vision processing," Mathematical Problems in Engineering, vol. 2011, Article ID 416963, 12 pages, 2011.

[22] X.-H. Yang, B. Wang, S.-Y. Chen, and W.-L. Wang, "Epidemic dynamics behavior in some bus transport networks," Physica A, vol. 391, no. 3, pp. 917-924, 2012.

[23] S. Chen, Y. Wang, and C. Cattani, "Key issues in modeling of complex 3D structures from video sequences," Mathematical Problems in Engineering, vol. 2012, Article ID 856523, 17 pages, 2012.

[24] M. Li, S. C. Lim, and S. Chen, "Exact solution of impulse response to a class of fractional oscillators and its stability," Mathematical Problems in Engineering, vol. 2011, Article ID 657839, 9 pages, 2011.

[25] K. S. J. Pister, J. M. Kahn, and B. E. Boser, "Smart Dust: wireless networks of millimeter-scale sensor nodes," Electronics Research Laboratory Research Summary, 1999.

[26] Crossbow Technology, http://www.xbow.com/.

[27] TinyOS, http://webs.cs.berkeley.edu/tos/.

[28] N. Cheraghi, M. J. Riley, and F. Taheri, "A novel approach for detection of damage in adhesivelybonded joints in plastic pipes based on vibration method using piezoelectric sensors," in Proceedings of the International Conference on Systems, Man and Cybernetics, pp. 3472-3478, October 2005.

[29] N. Cheraghi and F. Taheri, "A damage index for structural health monitoring based on the empirical mode decomposition," Journal of Mechanics of Materials and Structures, vol. 2, no. 1, pp. 43-62, 2007.

[30] L. Zhou, O. Yang, and J. Ou, "Design and analysis of laboratory health monitoring model of a long-span cable-stayed bridge," in Proceedings of the 20th National Compilation of Vibration and Noise of High Technology and Application Conference, pp. 57-66, 2007. 


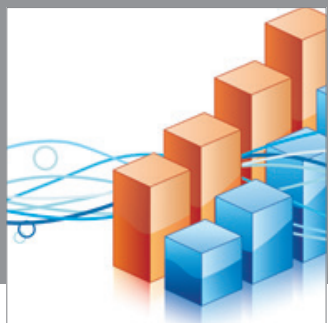

Advances in

Operations Research

mansans

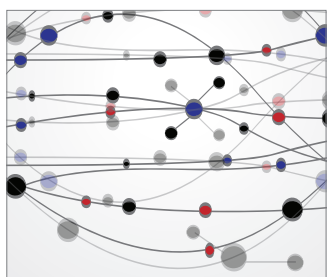

The Scientific World Journal
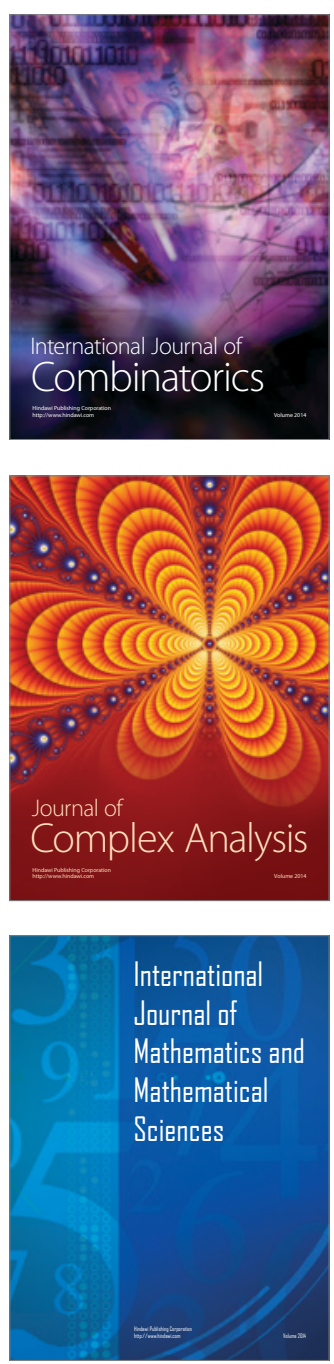
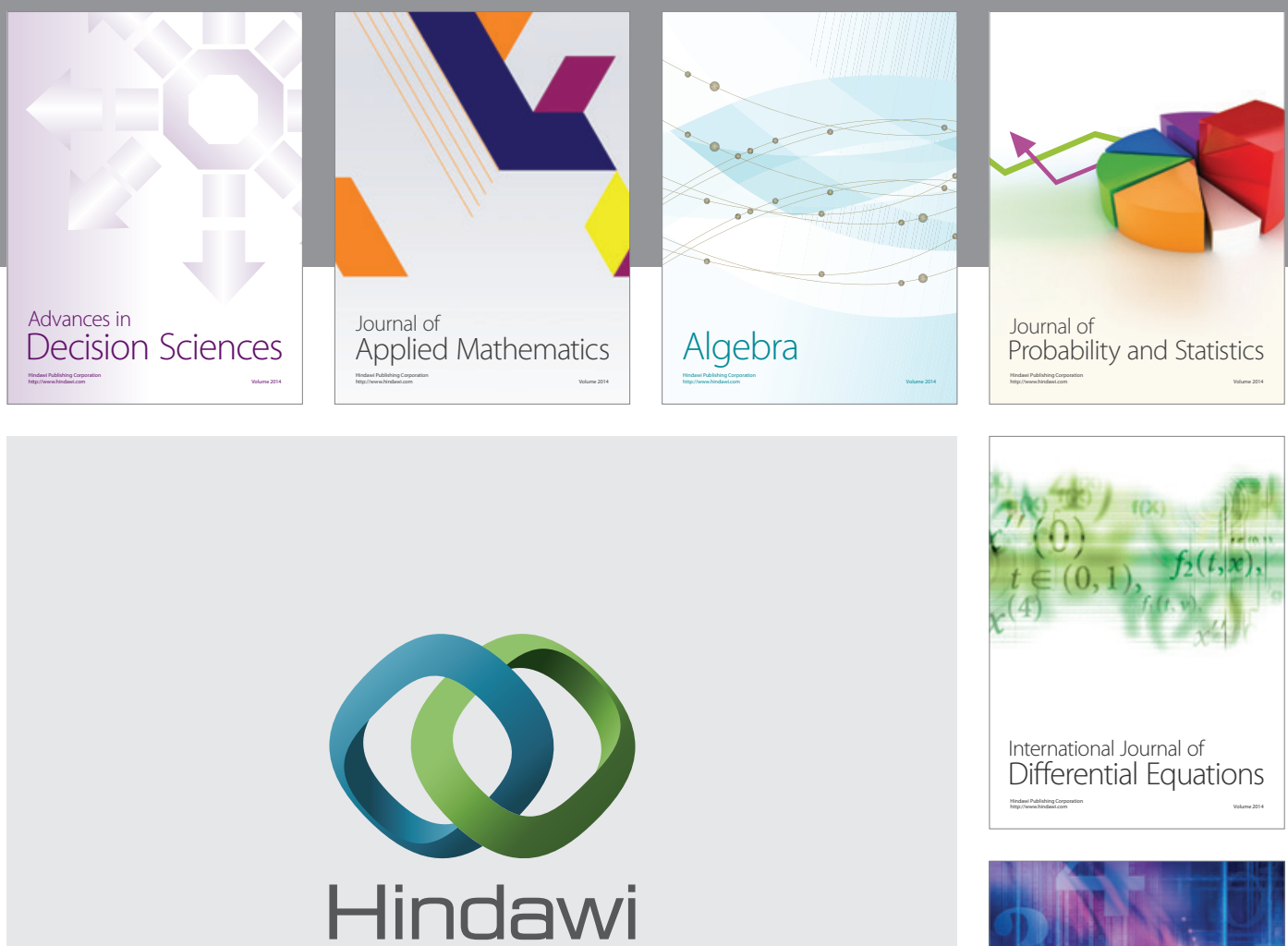

Submit your manuscripts at http://www.hindawi.com
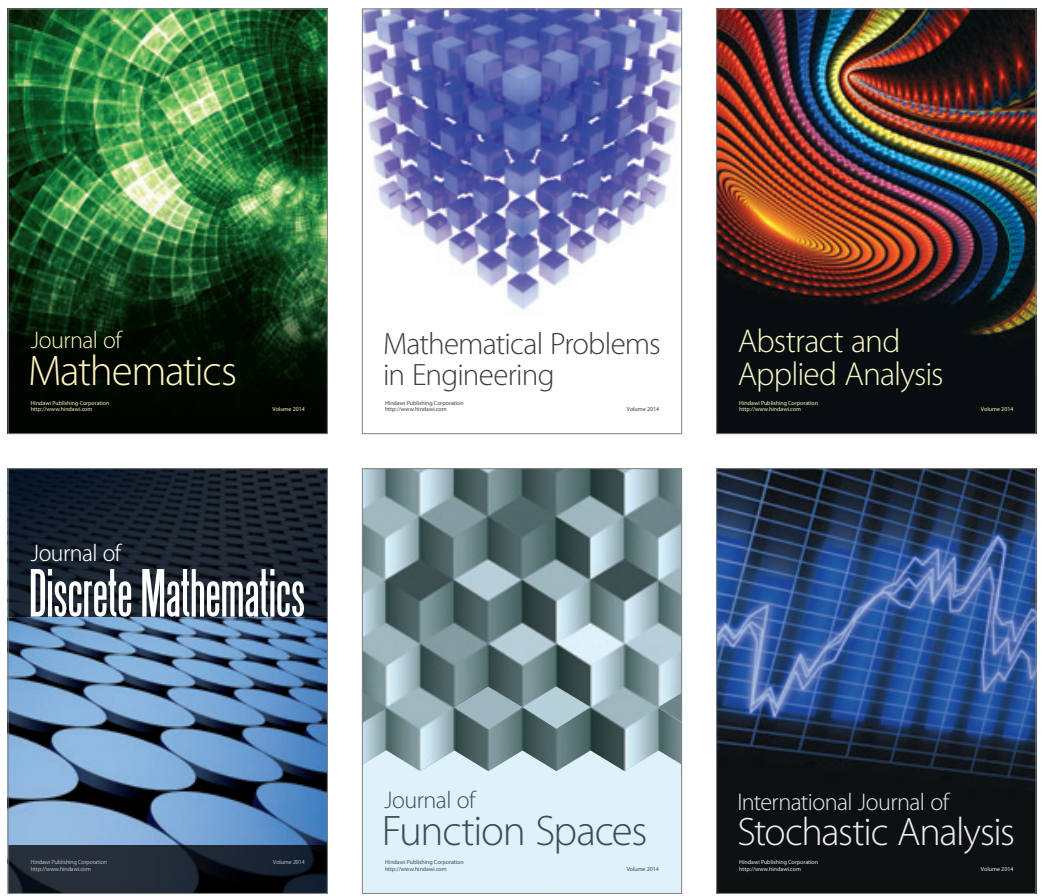

Journal of

Function Spaces

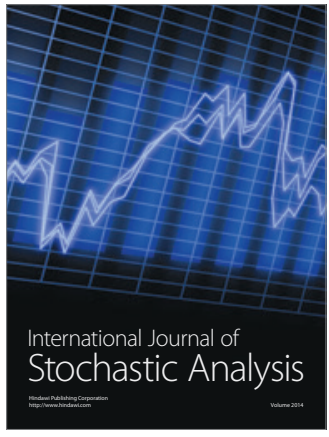

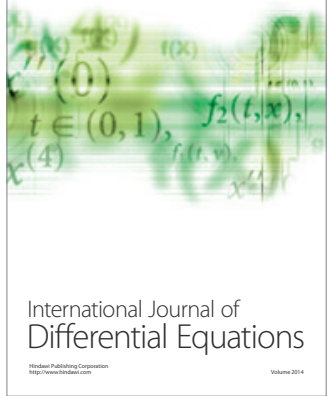
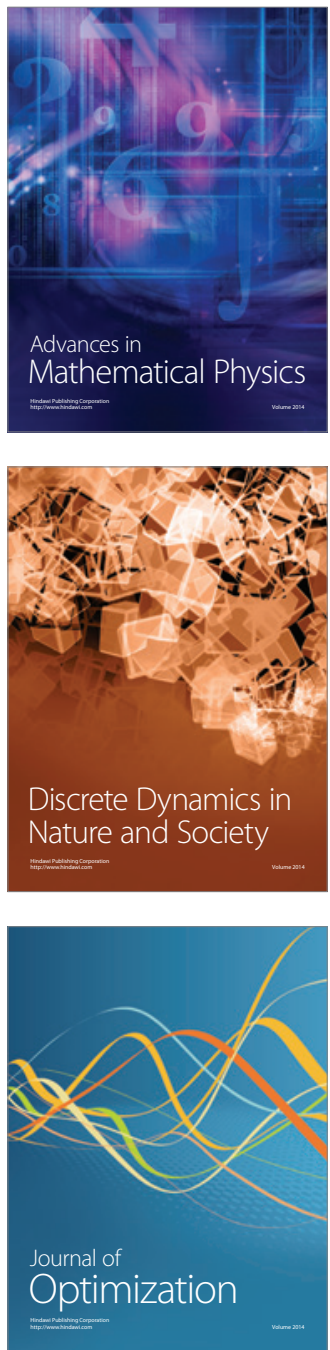